\title{
Gossypiboma Mimicking Gastrointestinal Stromal Tumor Causing Intestinal Obstruction: A Case Report
}

\author{
Yurika Kawamura ${ }^{a} \quad$ Naotaka Ogasawara $^{a}$ \\ Sayuri Yamamoto ${ }^{\text {a }}$ Makoto Sasaki $^{\text {a }}$ Naohiko Kawamura $^{a}$ \\ Shinya Izawa ${ }^{a}$ Yuji Kobayashi ${ }^{a}$ Seiji Kamei ${ }^{b}$ \\ Masahiko Miyachi $^{c} \quad K^{\prime}$ unio Kasugai ${ }^{a}$ \\ Departments of ${ }^{\mathrm{a}}$ Gastroenterology, ${ }^{\mathrm{b}}$ Radiology and ${ }^{\mathrm{c}}$ Surgery, Aichi Medical \\ University School of Medicine, Nagakute, Japan
}

\section{Key Words}

Small intestine $\cdot$ Obstruction $\cdot$ Textiloma $\cdot$ Gauze $\cdot$ Foreign body

\begin{abstract}
A 41-year-old woman was admitted to our hospital with abdominal pain that developed about 1 year after a Cesarean section. Pelvic computed tomography (CT) revealed diffuse dilation of the small intestine with fluid shadows and a pelvic tumor $55 \mathrm{~mm}$ in diameter. The density of the tumor, which was not enhanced by intravenous contrast medium, was diffuse and similar to that of muscular tissue, whereas the density of a capsule surrounding the mass was relatively high. T1- and T2-weighted pelvic magnetic resonance imaging (MRI) of the tumor revealed the same diffuse low-intensity signals as muscular tissue, and diffuse high-intensity signals, respectively. The CT and MRI findings were consistent with those of a gastrointestinal stromal tumor (GIST) causing ileus of the small intestine. As inserting an ileus tube did not improve her symptoms, the patient was scheduled for tumor resection. The operative findings revealed a hard, solid tumor adhering to the surrounding small intestine. The macroscopic findings revealed that the tumor consisted of layers of stratified gauze surrounded by a thick granulomatous wall. The gossypiboma was considered to have originated from gauze that had been left behind after the Cesarean section. If a patient has a history of surgery, the possibility of gossypiboma should be considered when CT or MRI findings indicate features of GIST.
\end{abstract}




\section{Introduction}

Retained surgical gauze (sponge) is called a gossypiboma or textiloma. The term gossypiboma is derived from a combination of the Latin word 'gossypium' for 'cotton' and the Swahili word 'boma' for 'place of concealment' [1]. Gossypiboma is a rare tumor caused by gauze fibers retained after surgery. Several gossypibomas after orthopedic [2], abdominal [3, 4] or plastic surgery [1] have been described. Gossypiboma in the abdominal cavity after surgery is a serious but avoidable complication. Despite measures taken by surgeons, foreign bodies continue to be retained after intra-abdominal or pelvic surgery $[5,6]$. The usual responses to retained surgical gauze are abscess formation with an exudative inflammatory reaction or aseptic mass development with a fibrotic reaction [7]. Patients develop symptoms of abdominal pain, nausea, vomiting, anorexia and weight loss resulting from an obstructive or malabsorptive type syndrome caused by multiple intestinal fistulas or intraluminal bacterial overgrowth. Gossypibomas are difficult to diagnose because they can mimic benign or malignant soft tissue tumors in the abdomen and pelvis and because the symptoms are usually nonspecific and can arise years after surgery $[5,6]$. Here we describe a patient with abdominal gossypiboma mimicking a gastrointestinal stromal tumor (GIST) that caused intestinal obstruction. Distinguishing gossypiboma from GIST before surgery by computed tomography (CT) and magnetic resonance imaging (MRI) is difficult.

\section{Case Report}

A 41-year-old woman underwent a Cesarean section in 2009. The Cesarean section was an emergency operation, and the patient was discharged after 11 days without complications. At that time, her weight was $56 \mathrm{~kg}$ and height $155 \mathrm{~cm}$ (body mass index 23.3), and she had no other risk factor except for the Cesarean section. She developed intermittent abdominal pain in September 2010. However, no symptoms of nausea, vomiting, anorexia or diarrhea were evident. The abdominal pain soon worsened and she was admitted to our hospital.

Physical examination revealed a tender lower abdomen and hyperactive bowel sounds. Rectal findings were normal. Laboratory investigations revealed white blood cells $7,400 / \mathrm{mm}^{3}$, C-reactive protein $0.61 \mathrm{mg} / \mathrm{dl}$, hemoglobin $11.4 \mathrm{~g} / \mathrm{dl}$, total protein $6.0 \mathrm{~g} / \mathrm{dl}$ and no abnormalities in liver and renal function tests. Abdominal and pelvic CT (fig. 1) revealed diffuse dilation of the small intestine with fluid shadows and a pelvic tumor $55 \mathrm{~mm}$ in diameter, but no thickening of the small intestinal wall, dilation of the large intestine or ascites. The density of the main tumor content was diffuse and low and similar to that of muscular tissue (fig. 1a), whereas that of a surrounding capsule was relatively high. Intravenous contrast medium did not enhance the main content of the mass, but slightly enhanced the surrounding capsule (fig. 1b).

The patient underwent abdominal and pelvic MRI examinations to obtain more information about

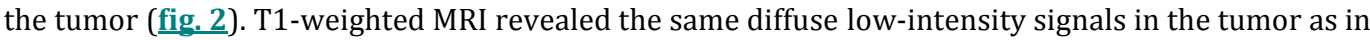
the muscular tissue (fig. 2a), and T2-weighted MRI revealed diffuse high-intensity signals indicating diffuse tumor necrosis as well as an irregular wall with low-intensity signals like muscular tissue (fig. 2b). The CT and MRI findings indicated a diagnosis of GIST with diffuse necrosis and the likelihood that mechanical ileus had caused the tumor. An ileus tube was inserted into the small intestine through the patient's nose to improve the ileus. However, an abdominal X-ray showed that the ileus did not improve and her symptoms persisted. Therefore, she underwent surgery to release the ileus and to resect the tumor 4 days after admission.

The operative findings revealed a hard, solid mass adhering to the surrounding small intestine. The tumor was peeled off from the small intestine and removed, together with the part of the small intestine that tightly adhered to the tumor. The ileus disappeared and she was discharged on 
postoperative day 7. The tumor consisted of layers of stratified gauze surrounded by a thick granulomatous wall ( $\underline{\text { fig. } 3}$ ). It was finally diagnosed as gossypiboma originating from gauze that had been left behind after the Cesarean section in 2009.

\section{Discussion}

Despite implementing many preventive measures during surgery, retained gauzes or sponges remain a major problem. Gossypiboma occurs in 1 among 100-3,000 of all surgical investigations and in 1 among 1,000-15,000 intra-abdominal operations, especially under conditions of busy surgical fields, emergencies, unplanned changes in procedures and a high body mass index $[3,8]$. In our patient, body mass index was within normal limit, and the emergency operation might have caused the retained gauze. The possibility of a retained foreign body should be considered in the differential diagnosis of any postoperative patient who presents with pain, infection or a palpable mass. A retained sponge may lead to the pathological formation of foreign body granuloma due to an aseptic fibrous response, or to an exudative reaction that results in abscess formation [9]. The former can have adhesions, encapsulation and eventually granuloma formation. However, the latter usually occurs early during the postoperative period and can involve secondary bacterial contamination resulting in various fistulas $[5,9]$.

Plain abdominal radiography, sonography, CT and MRI scanning are useful diagnostic tools $[3,8,10]$. The manifestations of gossypibomas on CT can change according to the locations and chronicity of the retained fabrics and the types of physiological reactions that they cause. A retained sponge on CT images is typically visualized as a well-defined soft tissue mass, often with a whorled or spongiform pattern with gas bubbles [11]. Lamellar, high-density areas also represent the sponge itself $[12,13]$. Calcification of the wall of the mass can be identified on CT scans [3]. Signal intensity on MRI may vary according to histological composition, stage and fluid content of the tumor. A retained sponge typically appears on MRI as a soft tissue mass with a thick, well-defined capsule and with complex mixed signal intensity similar to the whorled appearance on T2-weighted images. T2-weighted MRI also typically shows a folded fabric appearance within a cystic mass.

However, CT and MRI did not uncover any of these typical findings of gossypiboma in our patient. The CT findings of our patient indicated well-defined, low-density soft tissue without a whorled texture, spongiform pattern, lamellar high-density areas or calcification of the wall of the mass. The abdominal entity was visualized as a soft tissue mass with a thick, well-defined capsule with mixed signal intensity on T2-weighted MRI. However, T2-weighted MRI showed neither a folded fabric appearance within the cystic mass nor a whorled appearance. We therefore diagnosed the mass based on the CT and MRI findings as a GIST originating from the abdominal mesenterium or small intestine.

Most GISTs appear on CT images as well-defined masses with attenuation that varies according to size. Small lesions that are usually benign tend to be well-defined and relatively homogeneous [14]. Larger lesions can have well- or ill-defined margins and inhomogeneous density on both unenhanced and contrast-enhanced scans [14]. Peripheral enhancement correlates with central hemorrhage, necrosis or cyst 
formation and peripheral areas of viable tumors [14]. Solid portions of tumors typically show low signal intensity on T1-weighted MRI, and intermediate to high signal intensity on T2-weighted images according to fluid content [14]. An obviously high signal on T2-weighted MRI should be considered to be indicative of the central hemorrhage, necrosis or cyst formation associated with GIST [14]. The features of the mass in our patient mimicked those of GIST and she was thus scheduled for surgical tumor resection. In addition, the obstruction of the small intestine induced by the mass required emergency surgery. To the best of our knowledge, two reports have described gossypibomas as the cause of small intestinal obstruction [10,15], so we considered that our case was relatively infrequent. Although gossypiboma is rare in routine clinical practice, it should be considered in the differential diagnosis of acute mechanical intestinal obstruction when patients have undergone previous surgery. The best approach to the prevention of this condition is the meticulous count of surgical materials, a thorough exploration of surgical sites at the conclusion of procedures and the routine use of surgical textile materials impregnated with a radio-opaque marker. If a patient has a history of previous surgical procedures, the possibility of gossypiboma should be considered when CT or MRI findings indicate features of GIST.
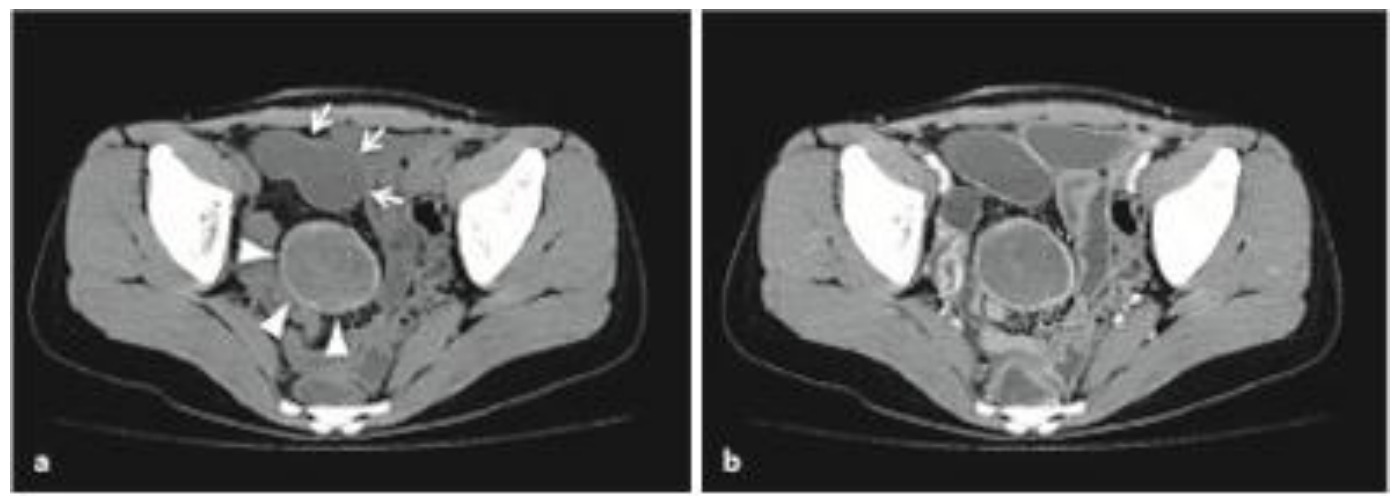

Fig. 1. Pelvic CT findings. a The pelvic tumor $55 \mathrm{~mm}$ in diameter with surrounding capsule has diffuse low density similar to that of muscular tissue, and the surrounding capsule has relatively high density (arrowheads). Diffuse dilation of the small intestine and fluid shadows are also evident (arrows). b The surrounding capsule is slightly enhanced by intravenous contrast medium, whereas main contents are not. 


\begin{tabular}{r|l|l|l}
$\begin{array}{r}\text { Case Reports in } \\
\text { Gastroenterology }\end{array}$ & $\begin{array}{l}\text { Case Rep Gastroenterol 2012;6:232-237 } \\
\text { DOI: 10.1159/000338833 }\end{array}$ & $\begin{array}{l}\text { Published online: } \\
\text { May 5, 2012 }\end{array}$ & $\begin{array}{l}\text { @ 2012 S. Karger AG, Basel } \\
\text { ISSN 1662-0631 } \\
\text { www.karger.com/crg }\end{array}$ \\
\hline
\end{tabular}
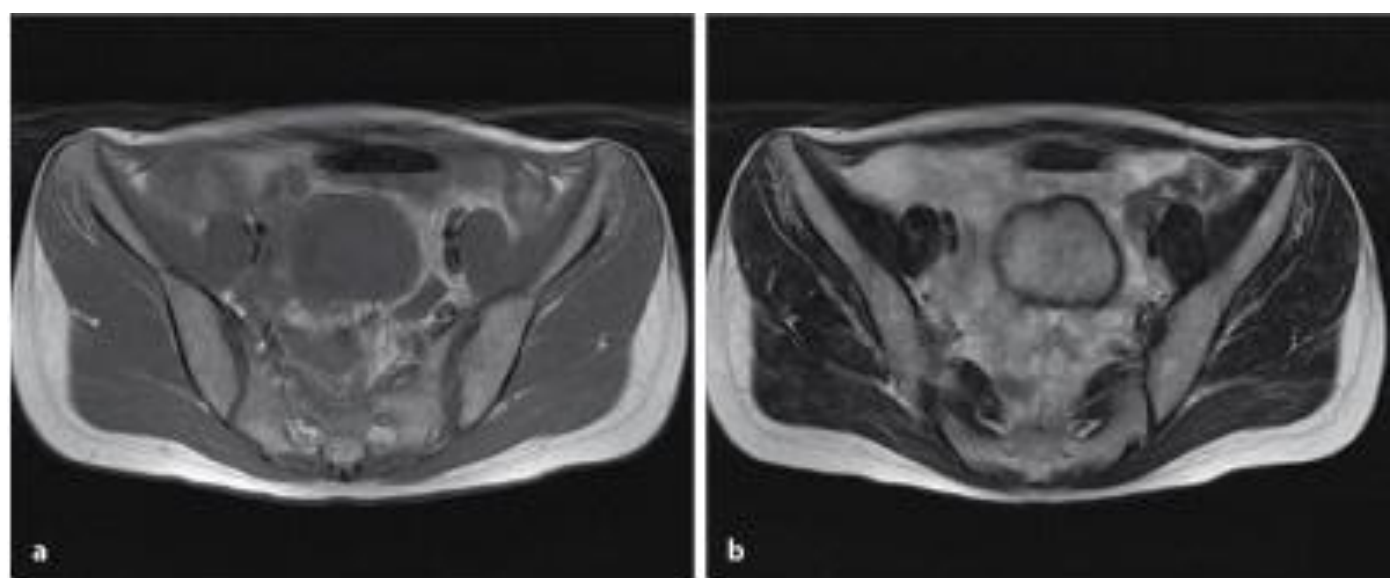

Fig. 2. Pelvic MRI findings. a T1-weighted MRI shows the tumor with surrounding capsule with same diffuse low-intensity signals as muscular tissue. b T2-weighted MRI shows diffuse high-intensity signals and irregular wall with low-intensity signals like muscular tissue.

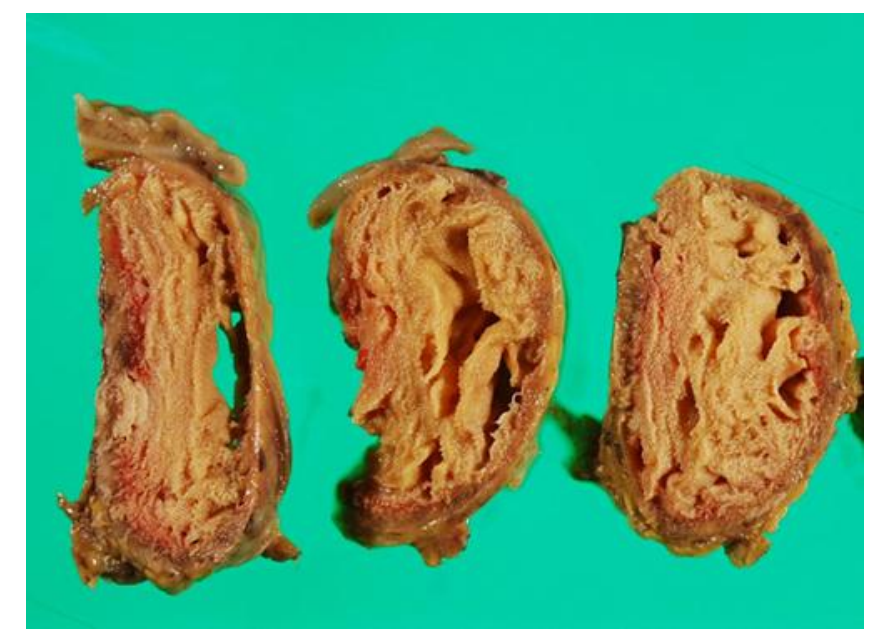

Fig. 3. Macroscopic findings of the resected tumor. Layers of stratified gauze forming the tumor are surrounded by a thick granulomatous wall.

\section{References}

1 Song SY, Hong JW, Yoo WM, et al: Gossypiboma after mandibular contouring surgery. J Craniofac Surg 2009;20:1607-1610.

2 Okten AI, Adam M, Gezercan Y: Textiloma: a case of foreign body mimicking a spinal mass. Eur Spine J 2006;15(suppl 5):626-629.

-3 Bani-Hani KE, Gharaibeh KA, Yaghan RJ: Retained surgical sponges (gossypiboma). Asian J Surg 2005;28:109-115.

4 Cevik I, Dillioglugil 0, Ozveri H, et al: Asymptomatic retained surgical gauze towel diagnosed 32 years after nephrectomy. Int Urol Nephrol 2008;40:885-888.

5 O'Connor AR, Coakley FV, Meng MV, et al: Imaging of retained surgical sponges in the abdomen and pelvis. AJR Am J Roentgenol 2003;180:481-489. 
6 Naik KS, Carrington BM, Yates W, et al: The post-cystectomy pseudotumour sign: MRI appearances of a modified chronic pelvic haematoma due to retained haemostatic gauze. Clin Radiol 2000;55:970-974.

7 Wan YL, Ko SF, Ng KK, et al: Role of CT-guided core needle biopsy in the diagnosis of a gossypiboma: case report. Abdom Imaging 2004;29:713-715.

8 Gawande AA, Studdert DM, Orav EJ, et al: Risk factors for retained instruments and sponges after surgery. N Engl J Med 2003;348:229-235.

9 Choi BI, Kim SH, Yu ES, et al: Retained surgical sponge: diagnosis with CT and sonography. AJR Am J Roentgenol 1988;150:1047-1050.

$\checkmark 10$ Gencosmanoglu R, Inceoglu R: An unusual cause of small bowel obstruction: gossypiboma - case report. BMC Surg 2003;3:6.

11 Kalovidouris A, Kehagias D, Moulopoulos L, et al: Abdominal retained surgical sponges: CT appearance. Eur Radiol 1999;9:1407-1410.

12 Cheng TC, Chou AS, Jeng CM, et al: Computed tomography findings of gossypiboma. J Chin Med Assoc 2007;70:565-569.

13 Suwatanapongched T, Boonkasem S, Sathianpitayakul E, et al: Intrathoracic gossypiboma: radiographic and CT findings. Br J Radiol 2005;78:851-853.

14 Chourmouzi D, Sinakos E, Papalavrentios L, et al: Gastrointestinal stromal tumors: a pictorial review. J Gastrointest Liver Dis 2009;18:379-383.

15 Rumstadt B, Roshanaei N, Schilling D: Gossypiboma - the retained surgical towel (in German). Dtsch Med Wochenschr 2008;133:1673-1676. 